\title{
Tops are more salient than bottoms
}

\author{
KENNETH W. CHAMBERS \\ Kent State University, Kent, Ohio \\ MICHAEL K. MCBEATH \\ Arizona State University, Tempe, Arizona \\ DIANE J. SCHIANO \\ Interval Research Corporation, Palo Alto, California \\ and \\ ERIC G. METZ \\ Kent State University, Kent, Ohio
}

\begin{abstract}
Past research has verified that observers assume that objects are reliably oriented with respect to a gravitationally centered coordinate system. Observers also appear to attend more to specific parts of objects, like faces, that typically are closer to the top. In the present work, we explored whether or not observers have a generic bias to view tops as being more salient than bottoms. In three experiments, observers indicated whether random shapes appeared to be more similar to comparison shapes that shared identical tops rather than bottoms. Observers exhibited a reliable tendency to match figures with similarly shaped tops. Matching choice was also a function of global shape attributes such as axis of elongation or size. The findings are consistent with the notion that, in nature, tops tend to be the most visible part and to provide the best information with respect to important aspects of objects such as animal intentionality and artifact functionality.
\end{abstract}

\section{Regularities of Orientation}

One of the primary problems of pattern recognition is that there are so many viable interpretations of the threedimensional (3-D) shape of a given proximal stimulus. Even well-defined 3-D objects can produce an infinite number of two-dimensional projections on the retina. In previous research, we explored systematic biases, or simplifying assumptions, that the visual system may use to constrain this stimulus indeterminacy during object recognition (e.g., McBeath, Schiano, \& Tversky, 1997). We demonstrated that viewers effectively use "default" assumptions about likely stimulus orientation to help constrain object identity, perhaps in a preliminary stage of the object-recognition process. Specifically, we found a pervasive tendency for viewers to interpret filled random figures as projections of bilaterally symmetric 3-D objects (also see Hayward, 1998). Vertically symmetric figures were reliably interpreted as straight-on views of objects, typically facing directly toward the viewer. Asymmetric figures were interpreted as side or profile views. System-

This work was supported by a grant from Interval Research Corp. We wish to thank Karen Chambers and Karen McBeath, for helpful comments on drafts of this manuscript, and Susan Suffion, for her help with data collection. Correspondence concerning this article should be addressed to K. Chambers, 1621 College St., South Bend, IN 46628 (e-mail: kwc@mad.scientist.com).

-Accepted by previous editor, Myron L. Braunstein atic manipulation of degree of figural symmetry produced a continuum of interpreted orientations. Although bilateral symmetry is not a universally invariant characteristic of objects, it occurs with such regularity that it can be considered as a near-invariant property of objects which our visual system appears to use in defining orientation.

The assumption of bilateral symmetry is consistent with the ecological observation that, to a first approximation, most lifeforms and artifacts are bilaterally symmetric. Thus, in the world, degree of figural or outline symmetry can be an effective first-order cue constraining the indeterminacy of object orientation and identity. Similar computational algorithms for determining the shape of symmetric 3-D objects from single outline views have been demonstrated in recent work in computer pattern recognition (Hayward, 1998; Poggio, Gamble, \& Little, 1988; Terzopoulos, Witkin, \& Kass, 1987).

We also examined a second regularity of orientation, the idea that most objects have a generally reliable upright orientation (Schiano, McBeath, \& Chambers, 1999). We verified that viewers assume a gravitationally centered coordinate system in which the tops of objects are oriented upwards. This further constrains the stimulus indeterminacy problem. Our work supports the idea that even when the effects of axis of symmetry and elongation are separated out under controlled conditions, viewers still show a consistent bias to assume that the top of a stimulus figure is also the intrinsic top of the interpreted object. The assumption of a reliable top-up orienta- 
tion is consistent with the ecological observation that, to a first approximation, most real-world transformations in vantage or orientation are lateral translations and rotations about a vertical axis. In other words, as we move about, we often experience differing lateral views of objects and much less often experience views in which we spin above or below objects. Many studies support the idea that rotations about the horizontal axis lead to more unnatural vantages (Rock, 1973; Thompson, 1980; Yin, 1969). In short, objects are assumed to be oriented "right-side up," or "top up."

In the present work, we explored a third objectrecognition strategy that emerges from the previous two orientation-constraining heuristics. Given that we assume that objects are generally symmetric and upright, then, a certain part of an object should reliably be in the same location on independent viewings. If certain parts are more informative than others, it would be advantageous to attend to their locations. In the present work, we explored the idea that tops of objects were more informative than bottoms and therefore were perceived as being more salient.

\section{Tops Are More Salient}

There are many reasons to argue that there is something special about the tops of objects (e.g., Franklin \& Tversky, 1990). The top-to-bottom axis of our bodies and of most objects is correlated with the only asymmetric axis of the world, that of gravity. Heads, faces, and the perceptual apparati of people and most animals are on top. These areas are particularly useful for giving us information about identity, behavior, and intentions, and are often required for communication. The tops of objects and artifacts also tend to be more visible, accessible for manipulation, and informative about functionality. We typically view objects from above (Mamassian \& Landy, 1996). We cannot even see the bottoms of objects, because their tops get in the way and occlude the bottoms.

Tops are also likely to stand out because they are typically the better illuminated portion of objects. In our world, light typically comes from above, and, as has been known since at least the 18 th century, this can have a profound effect on our interpretation of visual stimuli. Rittenhouse (1780) demonstrated, in a very early report of a top lighting bias in what has become known as the crater illusion, that changing the direction of lighting can change a person's perception of indentations as being either concave or convex.

The concept of "up" also appears to have a special linguistic status. "Up" tends to be linguistically unmarked and cognitively more accessible than "down" (e.g., Clark, 1973; Clark \& Clark, 1977; Shepard \& Hurwitz, 1984). Phrases like "how high" or "how tall," as opposed to "how low" or "how short," are default terms for describing vertical measures. One of the more common metaphors used across cultures is "up is good," as in "things are looking up" (Lakoff \& Johnson, 1980).

All written languages and musical notations read top to bottom. Some read in rows and some in columns, some left-to-right and some right-to-left, but all begin at the top of the page and proceed downward. The tops of figures, alphanumeric symbols, and faces tend to have higher information content (Attneave, 1955; Yarbus, 1967). Viewers tend to scan such stimuli longer near the top and move from the top down (Bruce, 1988; Jolicoeur, Ingleton, Bartram, \& Booth, 1993; Yarbus, 1967). Television, video, and computer raster-scan displays are also universally designed to scan from top to bottom before jumping back up to the top.

Even abstract, nonrepresentational figures may be interpreted as having a "correct" right-side up orientation, implying an intrinsic top and bottom. Arnheim (1954) found significant agreement between observers on the correct upright orientation of abstract art. Four- and 5-yearold children, across cultures, regard abstract geometric shapes as having intrinsic tops (Antonovsky \& Ghent, 1964; Ghent, 1961). Winner, Dion, Rosenblatt, and Gardiner (1987) were able to easily teach observers that art appears more balanced if there is less mass in the top of the image than in the bottom. Yet they were unable to teach observers to view images as being more balanced if there was more mass in the left than in the right.

In summary, there is a great deal of evidence that tops of objects and of graphical representations are typically more meaningful and informative than bottoms, suggesting that we ought to give more weight to them. However, the existence of a generic "default" bias to regard the tops of shapes as being more salient in the absence of informative natural patterns has not been demonstrated well under empirically controlled conditions. Kerpelman and Pollack (1964) did make an attempt to address this question with children; but their stimulus set was very limited and biased to be more discriminable in the bottom half of the figures, and their results were ambiguous. We have designed a study that should provide a fairer test of this issue.

In the present study, we used the same randomly generated stimulus figures as those used in our earlier research on orientation biases in object identification (McBeath et al., 1997). If the tops of objects are generically more salient than their bottoms, this effect should emerge with indeterminate stimuli of this sort, which minimize confounds due to attention-drawing internal contours and meaningful content (e.g., features such as eyes). This approach provides a conservative test of the top-salience hypothesis, since separating out the effects of top location from its common correlates in real-world objects might substantially reduce the magnitude of the observed effect. The use of random shapes removes any practical function of a top-salience heuristic and serves as a more pure measure of bias.

Three experiments were performed to investigate the top-salience hypothesis. Experiment 1 provides a straightforward, unbiased test of top-salience predictions with controlled random stimuli in a perceptual matching task. Experiment 2 replicates and extends the perceptual-match test with a manipulation of degree of figural similarity. 


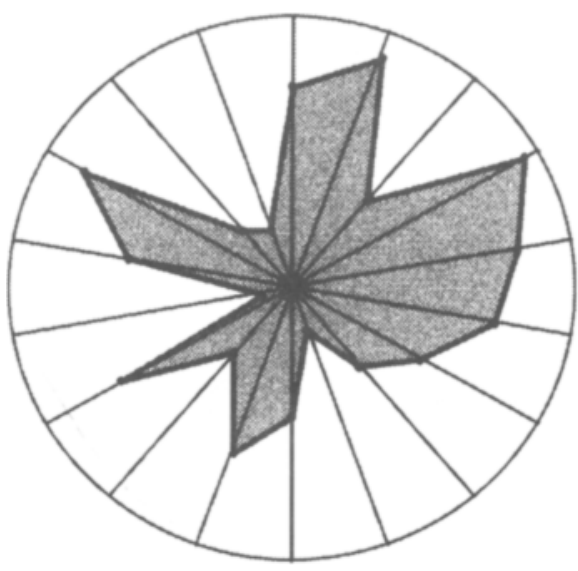

(a)

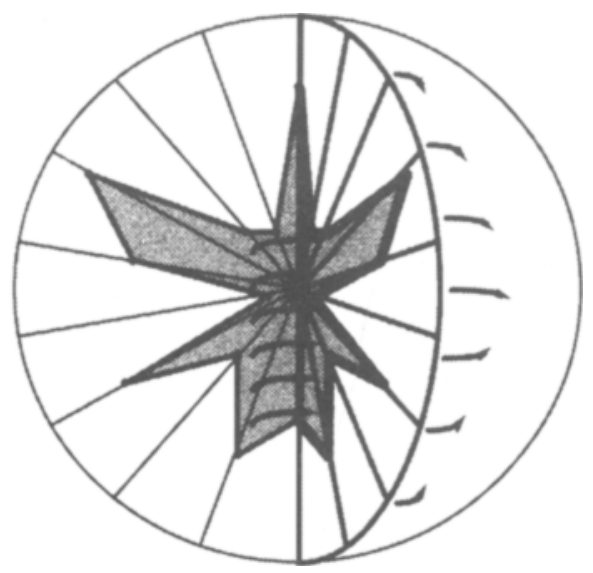

(b)

Figure 1. Method of stimulus figure generation. (a) Asymmetric figures: Random points along 18 equally spaced radii are connected and the center is filled in. (b) Symmetric figures: Half of an asymmetric figure is reflected about the vertical axis.

Experiment 3 uses a multidimensional scaling procedure with a free-sorting task to help distinguish additional global shape attributes that complement salience of the top when categorizing indeterminate figures.

\section{EXPERIMENT 1}

The experimental task was to indicate which of two comparison shapes better matched a test stimulus shape. In each pair of comparison figures, one figure had the same top as the test figure's and the other had the same bottom as the test figure's. The top-salience hypothesis predicts a bias to match the test figure with the comparison figure with the same top more often than with the comparison figure with the same bottom.

A symmetry manipulation was also included in this study, in order to expand on our earlier investigations of the role of vertical symmetry in perceiving outline figures (McBeath et al., 1997). Our previous work showed a strong bias to interpret indeterminate figures as upright vertically symmetric objects, with degree of figural symmetry directly determining perceived lateral vantage. However, if the primary role of figural symmetry is to serve as an initial cue to global orientation, no effect would be expected in the present similarity matching task, since both asymmetric and symmetric figures should be viewed as oriented "top up." Similarly, no interaction between symmetry and top salience would be predicted either. Thus, the strong and pervasive figural symmetry differences found in our work on figural interpretation were not expected to occur in this study. We therefore predicted an equally strong top-salience effect for asymmetric and symmetric shapes.

\section{Method}

Observers. Observers in all three experiments were Kent State University undergraduates who volunteered for extra credit in their general psychology course. All had normal or corrected-to-normal vision and were unaware of the hypotheses being tested. Fifty-eight observers participated in this experiment, in small groups of 2 to 12 people per test period.

Stimuli. The test figures used in all three experiments were 18 sided filled random polygons, previously used to demonstrate orientation biases in object identification (McBeath et al., 1997; Schiano, McBeath, \& Bruner, 1994). Asymmetric test figures were constructed by dividing a circle into equally spaced radii and randomly picking a point on each radius. The points were connected and the center filled in (see Figure 1a). To produce a symmetric test figure, half of the points of an asymmetric figure were reflected about a diameter (see Figure 1b) (after Farrell \& Shepard, 1981). A novel control half figure was added either to the top or the bottom of each test figure to form the comparison pairs (see Figure 2). Twenty symmetric and 20 asymmetric test figures were used, presented both in their original orientations and in their inverted orientations, resulting in 80 sets of perceptual-match figure trials.

Procedure. The observers were instructed to look at the figure in the middle of each row and to circle the figure on the right or the left that was the more similar one. They were told that there was no time limit, but to not perseverate about their choices. Between 10 and 25 min were typically required to complete the task.

\section{Results}

To determine whether the top was more salient than the bottom in this task, we computed the proportion of times a given half figure was chosen when it was presented in the top of a comparison figure compared with when it was presented in the bottom. Sample results are shown in Figure 3. The magnitude of the bias to favor top matches is computed by adding the "proportion same top picked" for the original and inverted orientations for each 


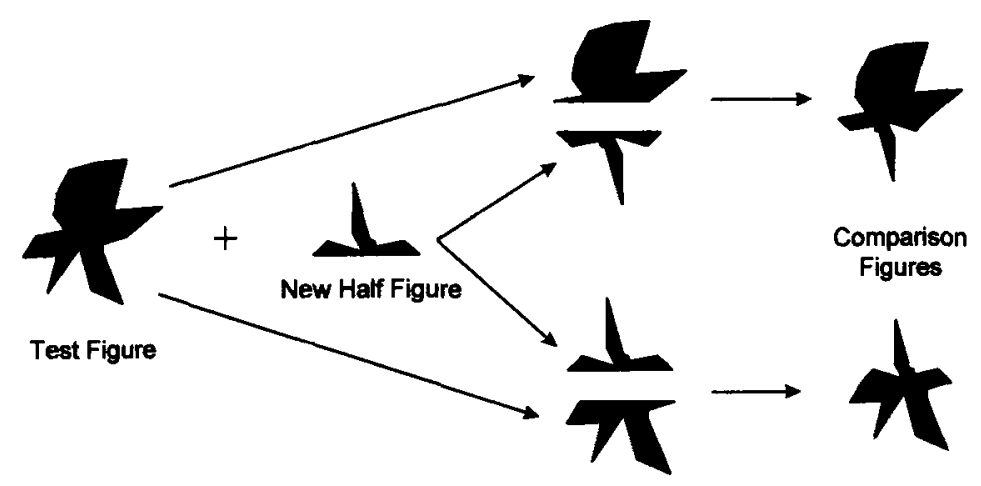

(a)

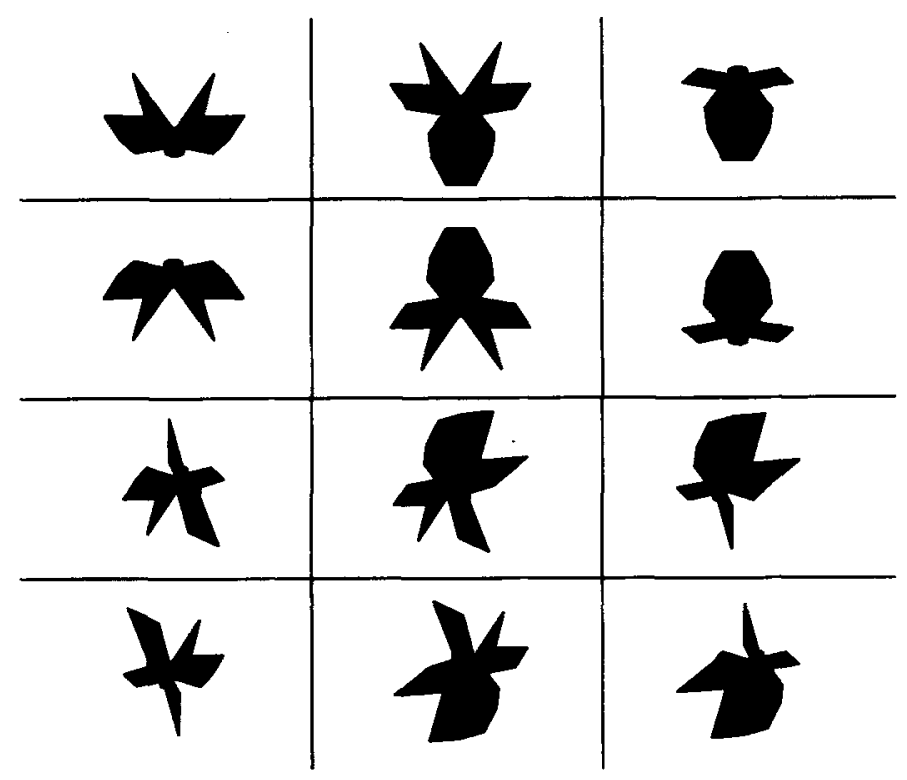

(b)

Figure 2. Stimuli used in Experiment 1. (a) Comparison figures are formed by combining a novel half figure with both the top and bottom of the test figure. (b) Examples of complete sets of figures for both original and inverted orientations of a symmetric and asymmetric test figure. The center column contains the test figures; the right and left flanking columns contain the "sametop" and "same-bottom" comparison stimuli.

test figure and comparing these sums with the expected chance value of 1 . We found that, overall, a given half figure was $8.9 \%$ more likely to be selected when it matched the top than when it matched the bottom. The effect was statistically different from chance when averaged across subjects $^{1}[t(39)=5.75, p<.001]$. There was no significant difference between the symmetric and asymmetric figures $[t(38)=1.86, p=$ n.s. $]$.

In addition to the bias to favor figures with matched tops, certain half figures tended to be more salient than others for perceptual matching, irrespective of whether they were on the top or the bottom. To measure this effect, we performed a chi-square test across subjects for each figure. We tested to see whether the frequency of choos- ing a comparison figure that shared a given half figure was independent of whether the figures were oriented normally or inverted (same top or same bottom). We found a significant orientation effect in 36 of the 40 figures, far more than expected by chance $(Z=34.37, p<$ $.001)$. This effect indicates that for each set of stimuli, one of the comparison figures was more likely to be chosen both when it was presented with the matching half figure in the top and with the matching half figure in the bottom. This supports the presence of salient shape attributes of figural similarity that are independent of a top-salience bias.

We also assessed whether or not the top-salience effect and the accidental features effect were independent of each 


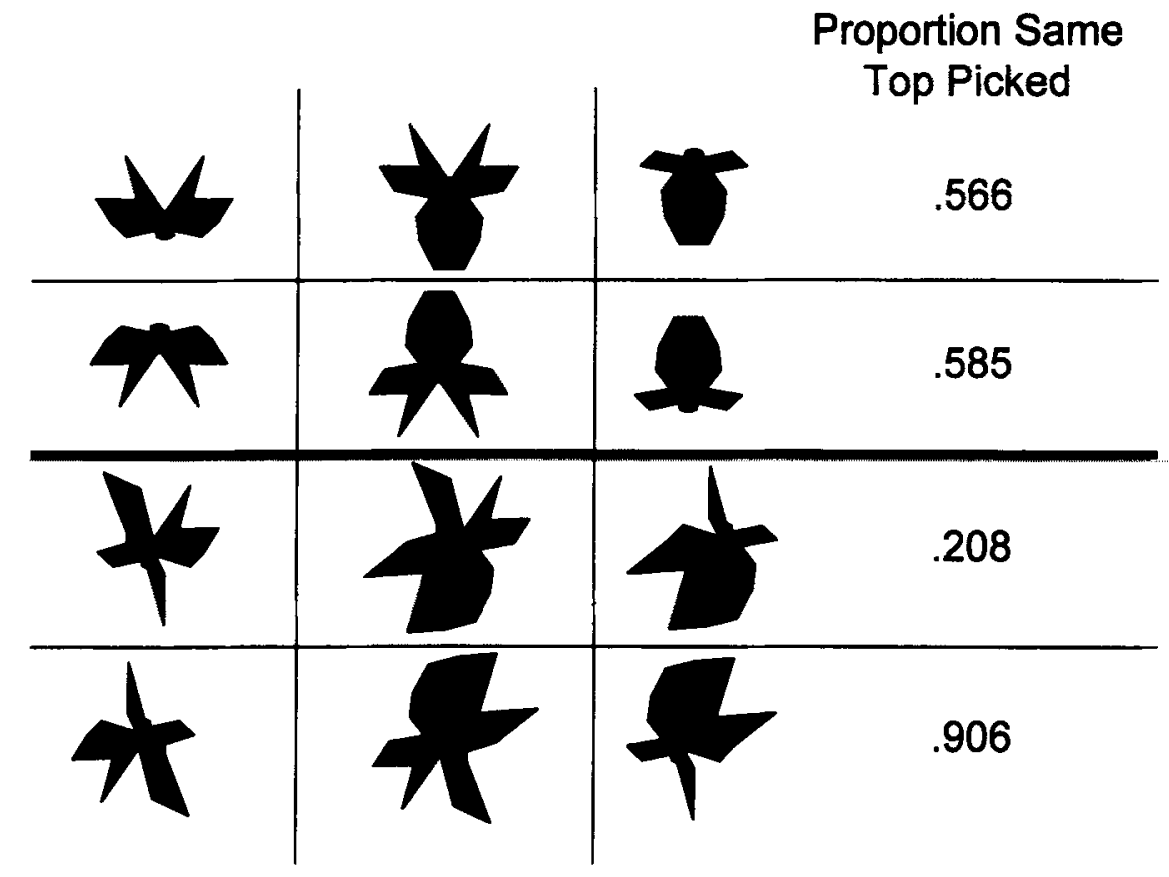

\begin{abstract}
Figure 3. Sample results from Experiment 1. The top two rows show the atypical response, where only the effect of top salience is operating. The bottom two rows show the far more typical situation, where the effects of both top salience and accidental shape features are operating. For any pair of test figures, when the sum of proportions of same top picked is greater than 1 , it indicates that the top was chosen more often than the bottom. The extent to which the proportions deviate from each other indicates the presence of accidental shape features.
\end{abstract}

other. Because these two effects are not additive, we did this by calculating the correlation between the magnitude of the top-salience effect for each figure with the magnitude of the accidental features effect for each figure. The effect of top salience and the effect of accidental features are independent, as is shown by the lack of a significant correlation between them for the $\mathbf{4 0}$ figures $(r=$ $.017)$.

\section{EXPERIMENT 2}

In the second experiment, we replicated and extended the investigation of the top-salience effect with slightly perturbed comparison figures. We explored the role of degree of figural similarity on perceptual matching by constructing comparison figures that varied in degree of similarity to the test figures. We also included trials in which the comparison figures had the same left or right shape as the test figure and could therefore serve as a lateral control condition. The top-salience hypothesis predicts a bias to match a test figure with a same-top, perturbed-bottom comparison figure more often than with a same-bottom, perturbed-top comparison figure. The left-right comparison serves principally as a control, although there is some evidence to support the idea that viewers trained to read left to right might have a left-side attentional bias that could conceivably make lefts more salient (Morikawa \& McBeath, 1992).
The perturbation manipulation provides a method for examining the effects of degree of figural similarity on top salience. A perturbation effect ought to depend on the relation between perturbation magnitude and object identity. With low levels of perturbation (near the threshold of perceived figural dissimilarity), comparison figures with one half perturbed should be perceived as so similar to the test figure as to be practically indistinguishable from it. Under these conditions, any top-salience effect should be small or nonexistent. With higher levels of perturbation, comparison figures should become progressively more dissimilar to the test figure and therefore more easily discriminable from it. Here the preference to match the comparison figure with the same top should become more noticeable. At some point, perturbation of the comparison figure becomes so large that the test and comparison figures may no longer appear to be from the same category. Here, again, the top-salience effect could level off or might even diminish. We predicted that, for our two levels of comparison-figure perturbation, the magnitude of the top-salience effect would differ. We also predicted that the figural symmetry would again fail to influence the top-salience effect.

\section{Method}

Observers. Fifty-four observers participated in Experiment 2 in small groups of 1 to 8 . All received credit in their general psychology courses and were naive as to the hypotheses being tested. All observers had normal or corrected-to-normal vision. 
Symmetric
$20 \%$ Perturbation

Symmetric $50 \%$ Perturbation

Asymmetric
$20 \%$ Perturbation

Asymmetric
$50 \%$ Perturbation

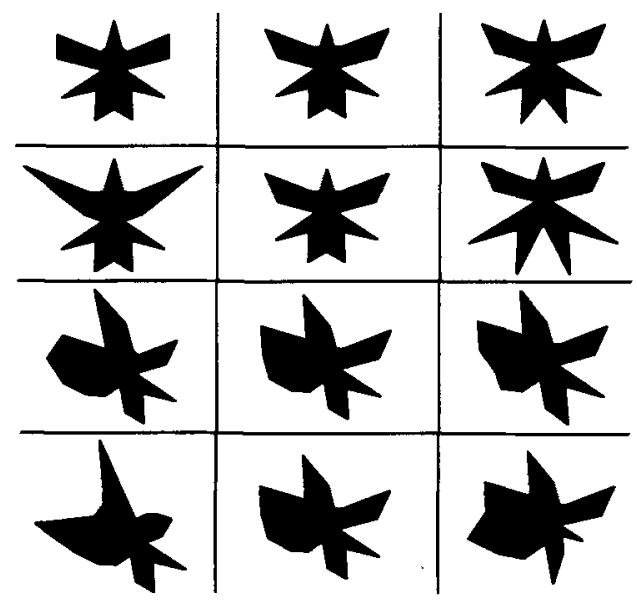

(a)

\section{Symmetric \\ $20 \%$ Perturbation}

\section{Symmetric $50 \%$ Perturbation}

\section{Asymmetric $20 \%$ Perturbation}

\section{Asymmetric $50 \%$ Perturbation}

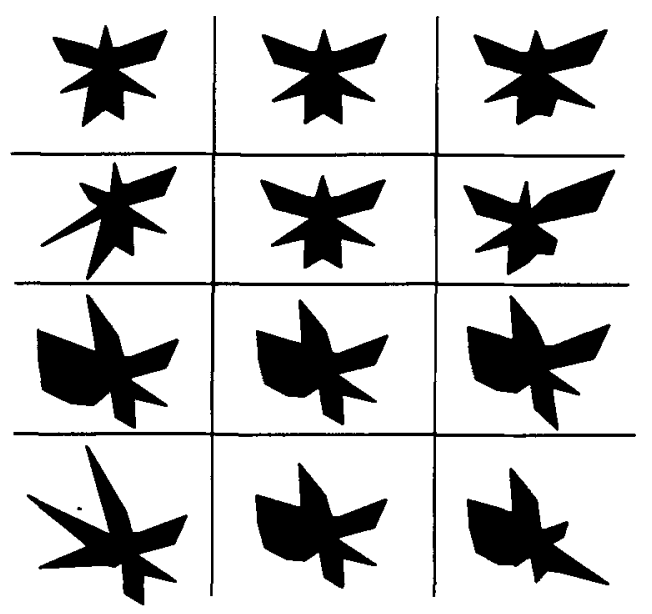

(b)

Figure 4. Examples of stimuli used in Experiment 2. In the center of each row is the base figure, on the right is the comparison figure with either the top or the left perturbed, and on the left is the comparison figure with either the bottom or the right perturbed. (a) Top/bottom stimuli. (b) Left/right stimuli.

Stimuli. The test stimuli used in this experiment were the same type of 18-sided outline polygons used in Experiment 1. However, this time, for every test figure, four pairs of comparison figures were generated. These included high and low perturbations of the top and bottom halves and high and low perturbations of the left and right halves (see Figure 4 for examples of stimuli). To produce the perturbations, the location of the points on the selected nine radii where moved by a percentage of their distance from the center, either closer to or further from the center. This percentage was randomly generated and was constrained to be within $20 \% \pm 5 \%$ of the total distance from the center in the small-perturbation condition and within $50 \% \pm 5 \%$ in the large-perturbation condition. Starting with 5 asymmetric and 5 symmetric test figures and their inversions, this resulted in a complete set of 80 triads of test and comparison figures. The full design consisted of 20 stimulus triads, 5 (base shapes) $\times 2$ (symmetric/asymmetric) $\times 2$ (low/high perturbation), displayed in four orientation conditions, 2 (top-bottom/ left-right) $\times 2$ (original/inverted). These were printed in random order, 5 per side of a page.

Procedure. Instructions to the observers were the same as in Experiment 1 . The typical time to complete the task was between 10 and $25 \mathrm{~min}$.

\section{Results}

Using the same dependent measure, combining original and inverted orientations, as in Experiment 1, we found that, on average, a given half figure was $10.0 \%$ more likely to be chosen when it was presented in the top $[t(19)=$ $4.43, p<.001]$. When the left-right figures are analyzed similarly, the figures with the same left were only $4.4 \%$ more likely to be chosen, a nonsignificant difference $[t(19)=1.45, p=n$.s. $]$. The perturbation manipulation 


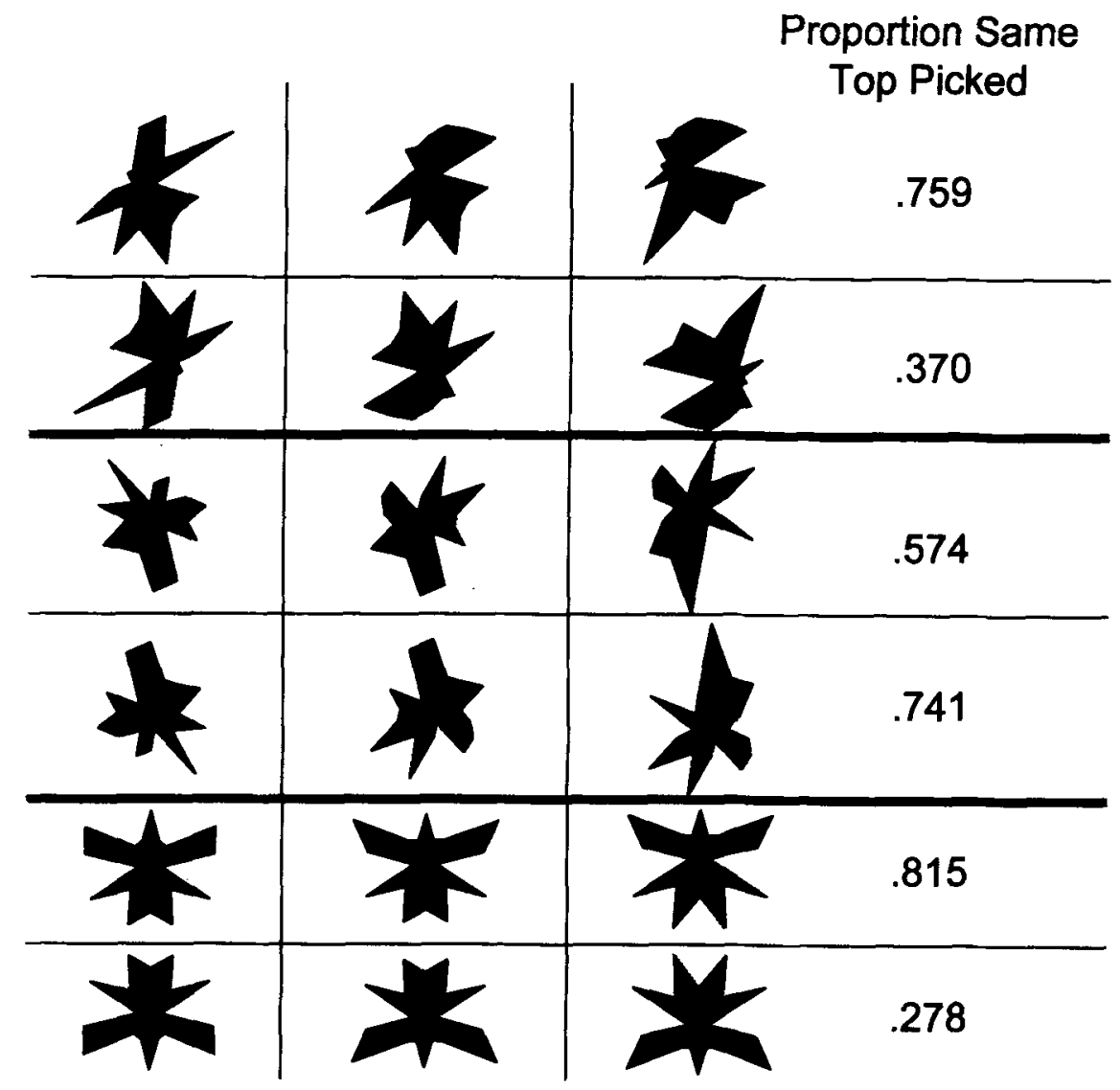

Figure 5. Sample results for three pairs of test figures in Experiment 2. The top and bottom pairs represent typical results; the middle pair shows the atypical pattern of responses. As in Experiment 1 , for any pair of test figures, a sum of proportions greater than 1 indicates that the same top was chosen more often than by chance.

changed the size of the top-salience effect as expected, although, with only two levels, we cannot fully specify the psychophysical continuum. The tendency to match comparison figures with the same tops was stronger in the figures with larger perturbations, $14.8 \%$, than in the figures with smaller perturbations, $5.2 \%[t(18)=2.39, p<.05]$. No significant difference was found between the asymmetric figures, $8.1 \%$, and the symmetric figures, $11.9 \%$ $[t(18)=0.84, p=$ n.s. $]$.

Using the same dependent measure as in Experiment 1, there was again a tendency for observers to match figures in part on the basis of accidental shape features regardless of whether the features were presented in the top or bottom $(Z=14.88, p<.001)$. This effect was found not only for the top-bottom manipulation, but also for the rightleft manipulation $(Z=14.88, p<.001)$. There was again no significant correlation between the top-salience effect and the accidental features effect for the 20 figures $(r=$ .167). Figure 5 shows sample results for several figures.

\section{Discussion}

Experiment 2 replicated the existence and magnitude of the top-salience effect found in Experiment 1. Fur- thermore, the null result with the left-right figures supports the unique salience of the top. The perturbation manipulation shows that degree of similarity of the test and comparison figures can influence the top-salience effect. Experiment 2 also confirmed the importance of accidental shape features. This finding suggests that there are strong global shape attributes that would be valuable to specify in order to better understand the occurrence of top salience.

\section{EXPERIMENT 3}

In the first two experiments, we found a strong effect of accidental features of the comparison shapes on judgments of similarity. Observers tended to match test figures with comparison figures that shared particular shape characteristics regardless of whether they were in the top or in the bottom. Our choice of random shapes instead of familiar objects may tend to accentuate such accidental effects. Still, the finding was robust enough for us to perform a third experiment to try to assess what common attributes the shape features possessed. We hypothesized that the shape features would reflect global orientation, 


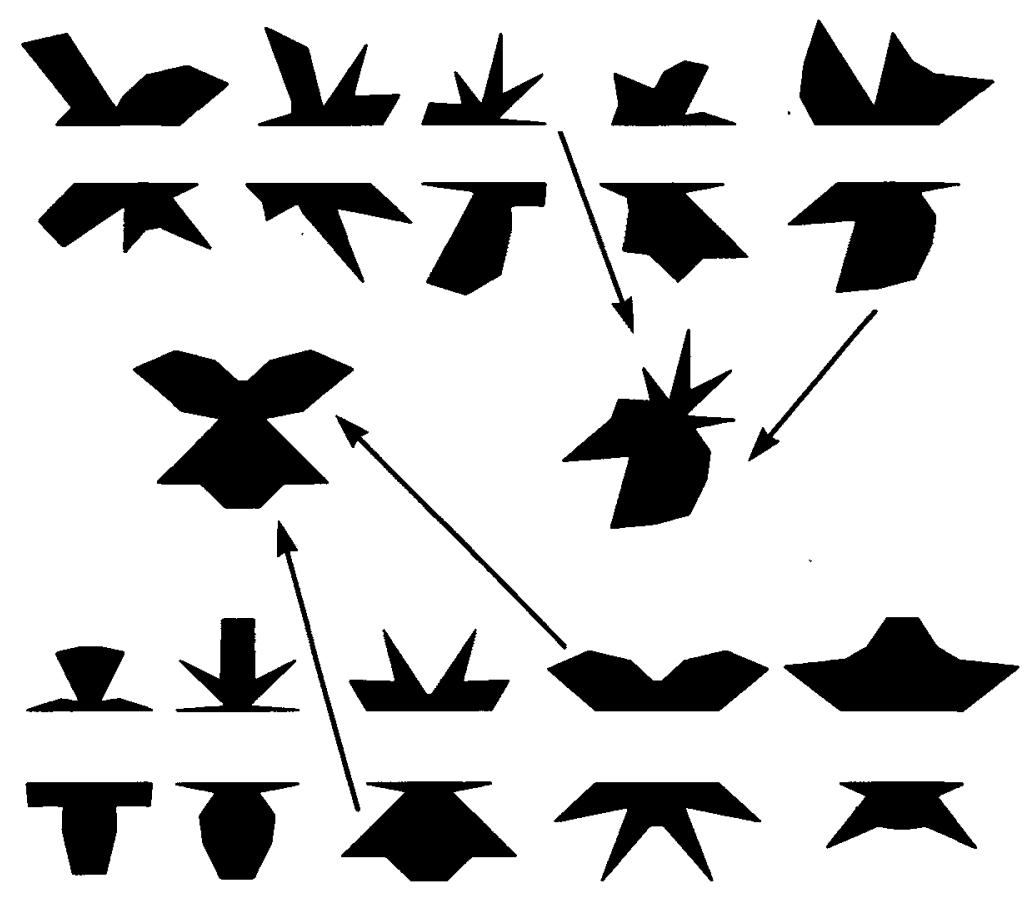

Figure 6. Figure construction for Experiment 3. On top is the complete set of asymmetric half figures used; on the bottom is the set of symmetric half-figures. All possible combinations of tops and bottoms were made. One symmetric and one asymmetric example are shown.

defining attributes that could be ascertained using a similarity sorting task. These might include attributes such as elongation and curvature. This experiment was designed to allow us to construct an attribute map using observers' groupings as the input for a multidimensional scaling solution. The results provided an idea of the underlying dimensions of the accidental shape features.

In Experiment 3, we took sets of 5 random top halves and 5 random bottom halves and made decks of cards containing all 25 possible combinations. Observers sorted the cards into groups on the basis of similarity. We anticipated that, owing to the top-salience effect, observers should group the figures more by tops than by bottoms. Yet presumably, once they began, the observers would be biased to continue using a consistent sorting strategy based largely on the results of the first few trials. Thus, the task of grouping could constrain degrees of freedom enough to prevent the emergence of a statistically significant top-salience effect. We anticipated that the principal variance would still reflect the salient global attributes that significantly influenced similarity judgments in Experiments 1 and 2.

\section{Method}

Observers. Seventy-seven naive undergraduate students participated in groups of 2 to 6 . All received course credit in their general psychology class for their participation. All had normal or corrected-to-normal vision.
Stimuli. The stimuli consisted of four sets of 25 laminated, white cards, each of which contained one black stimulus figure centered on the card and "Exp. 5a" in the upper left corner as an indirect indication of card orientation. The stimulus figures where constructed by taking a set of 10 random half figures, 5 designated as tops and 5 designated as bottoms, and making all possible combinations of tops and bottoms (see Figure 6). Four sets were made: both the original orientation and the inverted version using 18-sided random shapes that were either symmetric or asymmetric, as in Experiments 1 and 2.

Procedure. Each observer grouped two sets of cards, one symmetric and one asymmetric. Free-sorting task instructions were used, in which the observers were simply asked to group the cards that seemed to go together and told that there were no right or wrong groupings. Half of the observers were told to form five groups of five cards each; the other half were told to make as many groups of whatever size they wanted. The cards were thoroughly shuffled and dealt out in front of the observer so that he or she could see all of them at once. This allowed for a preview of the entire stimulus set and diminished the effects of early matches.

\section{Results}

As we speculated, an initial analysis of whether the subjects' groupings of the cards where based on the tops or bottoms of the figures did not directly confirm grouping according to the tops. The distribution of groupings was trimodal, with one proportion of observers making groupings consistent with matching the tops of the figures, a second proportion with groupings consistent with matching the bottoms, and a larger, third proportion with 


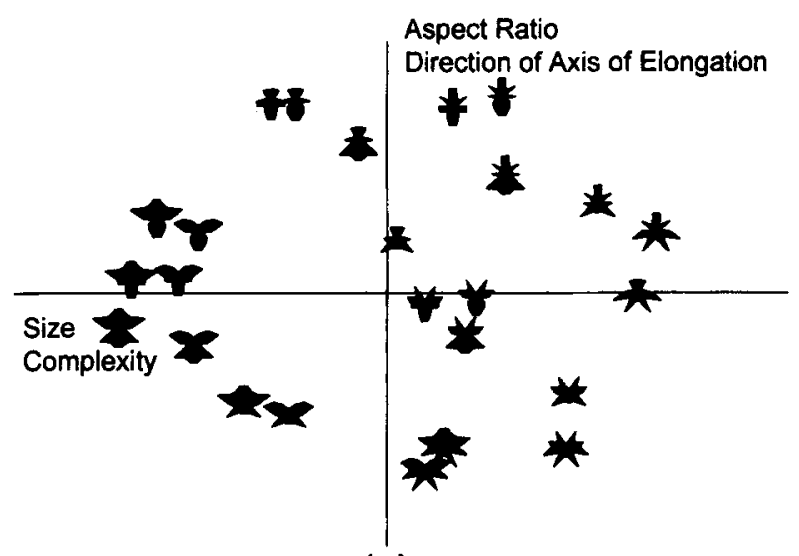

(a)

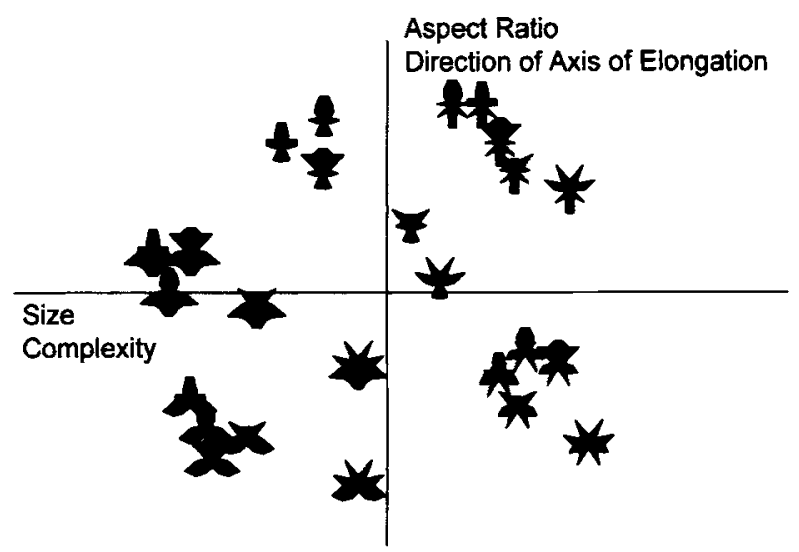

(b)

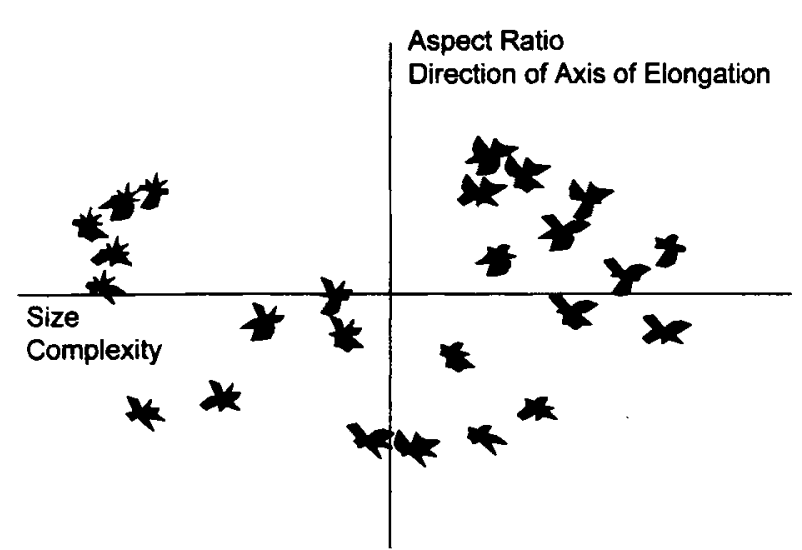

(c)

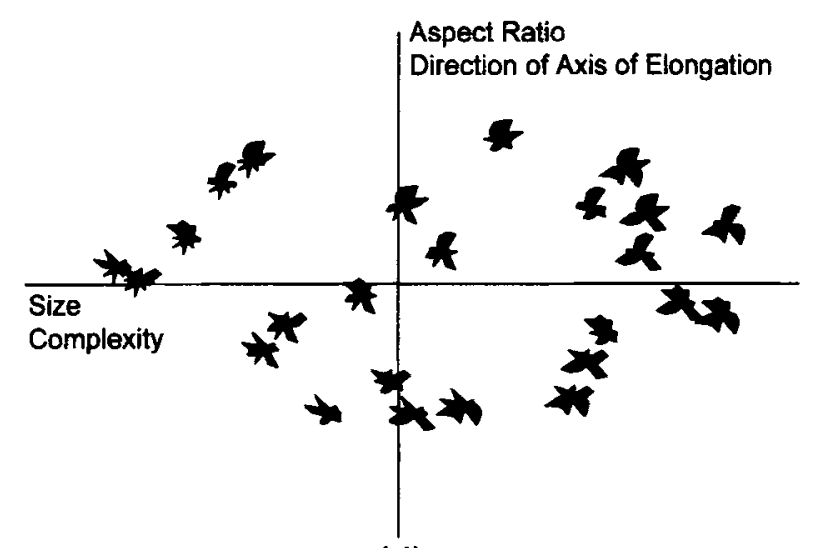

(d)

Figure 7. Multidimensional scaling solutions for Experiment 3. (a) Symmetric figures, original orientation. (b) Symmetric figures, inverted orientation. (c) Asymmetric figures, original orientation. (d) Asymmetric figures, inverted orientation. The two dimensions that emerged in all four conditions were (1) aspect ratio or axis of elongation and (2) size or complexity.

groupings that were not systematically biased one way or the other. The trimodal distribution is consistent with the use of a global strategy to stick with initial choice to match either tops or bottoms and is consistent with the occurrence of much less than $25 d f$ with respect to the number of matches. Such a reduction in degrees of freedom may well inhibit a significant top-salience effect from emerging in this type of analysis.

The second and principal analysis of this experiment was a multidimensional scaling procedure used to assess the major dimensions underlying similarity of features in the figures. We found no notable differences between the two sorting-instruction groups or between the two orientations of each of the symmetric and asymmetric sets of figures. The stimulus arrangement and dimensions appeared to be the same, independent of instruction and orientation. We therefore collapsed our solutions across instructions and rotated the different orientation solutions to match each other. The four resulting solutions are shown in Figure 7. The two main dimensions appear to be direction of axis of elongation or aspect ratio, shown on the vertical axis, and size or complexity, shown on the horizontal axis. The global orientation defining nature of the dimensions that emerged suggests that the accidental features that viewers attend to reflect basic level attributes used to ascertain generic orientations of figures. Such attributes appear to predispose the viewers' interpretations, helping to define such things as which direction is up in specifying the top of an object.

\section{GENERAL DISCUSSION}

In the first two experiments, two principal trends were demonstrated: (1) Observers judged that figures that shared a common top were more similar to each other than figures that shared a common bottom, and (2) some accidental shape features were particularly salient regardless of where in the figure they were presented. The first 
result is consistent with our hypothesis that the tops of objects are more salient than other parts of objects. This bias was confirmed even with the random, unnatural stimulus figures we used. The bias to give more weight to the tops of objects adds to the list of heuristics that help observers solve the stimulus indeterminacy problem in object recognition. This work supports the idea that people typically attempt to first identify the upper portions of objects. The findings can also account for small, but persistent, top-bottom effects found in other shapematching work (e.g., Wolfe \& Bennett, 1997). In a visual search task, Wolfe and Bennett had target figures in sets of distractor figures that had either the same top half or the same bottom half as the target figure. In all set sizes, their subjects were faster to find the target when it had a top that was different form the rest of the set.

Clearly, the top-salience bias was not the largest effect we.found, and there are limitations to it. First, it appears to apply to our dealings with individual objects but not necessarily scenes. In scenes, people typically tend to pay more attention to the bottoms of the configuration (Yarbus, 1967). The information at ground level is usually more important than the information in the sky. Graphs also may be processed the same way (Schiano \& Tversky, 1992; Tversky \& Schiano, 1989). Second, we also suggest that there is an issue of object size that interacts with this bias. When visiting Redwood National Park, most people no doubt look at the bottoms of the important objects. When people approach a house, they are probably more likely to notice the front door than the chimney. Thus, the bias we have documented is apt to be most apparent with objects that are roughly our size and smaller, for example, people, animals, and objects that we can easily manipulate.

The second principal finding is that particular features appear to be salient regardless of location. The third experiment quantified some of the underlying dimensions of the features that are most salient to observers. The dimension of axis of elongation is consistent with observers' being very aware of orientation in order to help constrain the stimulus indeterminacy. Particular sensitivity to orientation is consistent with the findings of previous work demonstrating that people assume objects are vertically symmetric and reliably ofiented with tops facing up (McBeath et al., 1997; Schiano et al., 1994).

These experiments provide direct, empirical evidence that humans do treat the tops of figures as more salient than the bottoms. They do this even with non-object-like random polygons, and presumably might do so even more strongly with real objects, for which it is likely the information in tops would actually be more informative. Knowledge of these findings may be useful in tasks ranging from graphic design to the development of computer software and virtual environments.

Moreover, these results are consistent with the idea that human object processing uses heuristics that are biased to favor stimuli likely to be present in nature. Ob- jects in the real world really do have a tendency to remain upright, and they really do have tops that are more informative. The tops of animals almost universally contain their heads and sensory apparati that indicate important aspects of behavior and intentionality. The tops of artifacts often contain the most important aspects with respect to accessibility or informativeness about their functionality. The occurrence of a generic bias to experience tops as being more salient reflects how our perceptual mechanisms have internalized these regularities of nature.

\section{REFERENCES}

ANTONOVSKY, H. F., \& GHENT, L. (1964). Cross-cultural consistency of children's preferences for the orientation of figures. American Journal of Psychology, 77, 295-297.

ARNHEIM, R. (1954). Art and visual perception. Berkeley: University of California Press.

ATtNEAVE, F. (1955). Symmetry, information, and memory for patterns. American Journal of Psychology, 68, 209-222.

BruCE, V. (1988). Recognising faces. Hove, U.K.: Erlbaum.

CLARK, H. H. (1973). Space, time, semantics, and the child. In T. E. Moore (Ed.), Cognitive development and the acquisition of language (pp. 27-63). New York: Academic Press.

Clark, H. H., \& Clark, E. V. (1977). Psychology and language: An introduction to psycholinguistics. New York: Harcourt Brace Jovanovich.

FarRell, J., \& ShePard, R. (1981). Shape, orientation, and apparent rotational motion. Journal of Experimental Psychology: Human Perception \& Performance, 7, 477-486.

Franklin, N., \& Tversky, B. (1990). Searching imagined environments. Journal of Experimental Psychology: General, 119, 63-76.

Ghent, L. (1961). Form and its orientation: A child's-eye view. American Journal of Psychology, 74, 177-190.

HaYWARD, W. G. (1998). Effects of outline shape in object recognition. Journal of Experimental Psychology: Human Perception \& Performance, 24, 427-440.

Jolicoeur, P., Ingleton, M., Bartram, L., \& Booth, K. (1993). Topbottom and front-behind decisions on rotated objects. Canadian Journal of Experimental Psychology, 47, 657-677.

Kerpelman, L. C., \& Pollack, R. H. (1964). Developmental changes in the location of form discrimination cues. Perceptual \& Motor Skills, 19, 375-382.

LAKOFF, G., \& Johnson, M. (1980). Metaphors we live by. Chicago: University of Chicago Press.

Mamassian, P., \& Landy, M. S. (1996, April). A prior for shape-fromcontour. Paper presented at the annual meeting of The Association for Research in Vision and Ophthalmology, Fort Lauderdale, FL.

McBeath, M. K., Schiano, D. J., \& Tversky, B. (1997). Threedimensional bilateral symmetry bias in judgments of figural identity and orientation. Psychological Science, 8, 217-223.

Morikawa, K., \& MCBeath, M. K. (1992). Lateral motion bias associated with reading direction. Vision Research, 32, 1137-1141.

Poggio, T., Gamble, E., \& LitTle, J. (1988). Parallel integration of vision modules. Science, 242, 436-440.

RitTenhouse, D. (1780, March). Explanation of an optical deception. Paper presented to the American Philosophical Society.

Rock, I. (1973). Orientation and form. New York: Academic Press

Schiano, D. J., McBeath, M. K., \& Bruner, K. W. (1994, November). Orientation-constraining heuristics for interpreting figures as 3-D objects. Paper presented at the 35th Annual Meeting of the Psychonomic Society, St. Louis.

Schiano, D. J., McBeath, M. K., \& Chambers, K. W. (1999). Random shapes are interpreted as silhouettes of bilaterally symmetric objects with tops on the axis. Manuscript in preparation.

SCHIANo, D. J., \& TVersky, B. (1992). Structure and strategy in encoding simplified graphs. Memory \& Cognition, 20, 12-20. 
ShePard, R. N., \& Hurwitz, S. (1984). Upward direction, mental rotation, and discrimination of left and right turns in maps. Cognition, 18, 161-193.

Terzopoulos, D., WITkIn, A., \& Kass, M. (1987). Symmetry-seeking models and 3D object reconstruction. International Journal of Computer Vision, 1, $211-221$.

Thompson, P. (1980). Margaret Thatcher: A new illusion. Perception, 9, 483-484.

Tversky, B., \& SChiano, D. J. (1989). Perceptual and conceptual factors in distortions in memory for graphs and maps. Journal of Experimental Psychology: General, 118, 387-398.

Winner, E., Dion, J., RoSEnBl,ATt, E., \& GARDINER, H. (1987). Do lateral or vertical reversals affect balance in paintings? Visual Arts Research, 13, 1-9.

Wolfe, J. M., \& Bennett, S. C. (1997). Preattentive object files: Shapeless bundies of features. Vision Research, 37, 25-43.
YARBUS, A. L. (1967). Eye movements and vision. New York: Plenum Press. YIN, R. K. (1969). Looking at upside-down faces. Journal of Experimental Psychology, 81, 141-145.

\section{NOTE}

1. The analyses in Experiments 1 and 2 were done by averaging across subjects and using the figures as the units of analysis. The total of 40 figures in Experiment $\mathbf{I}$ resulted in $d f=\mathbf{3 9}$ for the one-sample $t$ test and $d f=38$ for the independent-means $t$ test. The total of 20 figures in Experiment 2 resulted in $d f=19$ for the one-sample $t$ tests and $d f=18$ for the independent-means $t$ tests.

(Manuscript received June 2, 1997;

revision accepted for publication February 27, 1998.) 